Louisiana State University

LSU Digital Commons

Faculty Publications

Department of Mathematics

$1-1-2013$

\title{
An adaptive control design for 3D curve tracking based on robust forward invariance
}

\author{
Michael Malisoff \\ Louisiana State University \\ Fumin Zhang \\ School of Electrical and Computer Engineering
}

Follow this and additional works at: https://digitalcommons.Isu.edu/mathematics_pubs

\section{Recommended Citation}

Malisoff, M., \& Zhang, F. (2013). An adaptive control design for 3D curve tracking based on robust forward invariance. Proceedings of the IEEE Conference on Decision and Control, 4473-4478. https://doi.org/ 10.1109/CDC.2013.6760578

This Conference Proceeding is brought to you for free and open access by the Department of Mathematics at LSU Digital Commons. It has been accepted for inclusion in Faculty Publications by an authorized administrator of LSU Digital Commons. For more information, please contact ir@lsu.edu. 


\section{An Adaptive Control Design for 3D Curve Tracking based on Robust Forward Invariance}

Michael Malisoff

\begin{abstract}
We study a class of adaptive controllers for three dimensional (3D) curve tracking under control uncertainty and unknown control gains. Using robust forward invariance and a Lyapunov-Krasovskii functional, we prove input-to-state stable tracking and parameter identification under input delays and a class of polygonal state constraints. This yields predictable tolerance and safety bounds, and compensation of arbitrarily long input delays in the important case where the delays only occur in the speed measurements in the controls.
\end{abstract}

\section{INTRODUCTION}

This note gives 3D, input delayed analogs of our adaptive robust curve tracking and parameter identification work [8], which was limited to adaptive 2D dynamics without delays. Our main tools are our recent strict Lyapunov function and robust forward invariant approaches for nonadaptive 3D curve tracking systems from [9], combined with a new construction of Lyapunov-Krasovskii functionals.

Our work is motivated by our recent deployment of a team of marine robots in a search for pollution from the Deepwater Horizon oil spill [12], which used our 2D curve tracking methods from [6], [8]. The robotic curve tracking problem has a rich literature [3], [14], [17] and leads to cooperative control methods that track motion for multiple mobile robots; see [18] for applications to ocean sensing. See also [2], [13], [15], [16] for autonomous controls for drogues and robotic fish. However, previously reported results for 3D curve tracking based on nonstrict Lyapunov functions do not lead to input-to-state stability (ISS) [4] under input delays, and do not prove state constrained results that give predictable tolerance and safety bounds under the unknown control gains that prevail in real life marine robotic deployments [12]. The ISS property is a key robustness condition that quantifies the effects of uncertainty, by generalizing the usual uniform global asymptotic stability (UGAS) decay estimate; see Section $\mathrm{V}$ for precise definitions.

For the much simpler case of 2D adaptive dynamics, or for 3D nonadaptive dynamics, our Lyapunov and robust forward invariance set (RFIS) approaches from [6], [9] involved decomposing the state space into a nested sequence $\mathscr{S}_{1} \subseteq \mathscr{S}_{2} \subseteq \mathscr{S}_{3} \subseteq \ldots$ of sets, and then finding maximal disturbance sets $\mathscr{D}_{i}$ such that for each index $i$, all trajectories of the tracking dynamics starting in $\mathscr{S}_{i}$ for all disturbances

Malisoff is with Department of Mathematics, Louisiana State University, 303 Lockett Hall, Baton Rouge, LA 70803-4918, malisoff@1su.edu. Zhang is with the School of Electrical and Computer Engineering, Georgia Institute of Technology, 85 Fifth Street NW, Atlanta, GA 30332-0250, fumin@gatech.edu. Malisoff was supported by NSF Grant ECCS-1102348. Zhang was supported by ONR Grants N00014-08-1-1007 and N00014-09-11074, and NSF Grants ECCS-0841195, ECCS-0845333 (CAREER), ECCS1056253, and CNS-0931576.

\author{
Fumin Zhang
}

valued in $\mathscr{D}_{i}$ remain in $\mathscr{S}_{i}$ for all positive times. The $\mathscr{D}_{i}$ 's are maximal in the Hausdorff topology, so they give predictable tolerance and safety bounds that say how large the perturbations can be before the state constraint set $\mathscr{S}_{i}$ is violated. Another advantage of the RFIS method is that under standard conditions, UGAS to any equilibrium on any compact RFIS $\mathscr{S}_{i}$ implies ISS on that set, because any strict Lyapunov function has a bounded gradient on $\mathscr{S}_{i}$, and so is also an ISS Lyapunov function. Finally, the RFIS approach can handle input delays, by incorporating the effects of the delays into the perturbations and using the sets $\mathscr{D}_{i}$ to compute maximum allowable delays [6]. This gives a new approach to input delay compensation that is totally different from results such as [10], [11], [20] (which compute maximum allowable input delays that can be introduced into undelayed feedback stabilizers while maintaining stability) or [1], [5] (where the controllers are based on prediction or reduction but do not ensure that state constraints can be satisfied).

Although this note owes a lot to [9], extending [6], [8], [9] to 3D adaptive curve tracking and control gain identification under delays is challenging. We review the background from [9] in the next section. In Section III, we provide the key extension of our RFIS results from [6], [8], [9] to the 3D case, and in Section IV we explain how RFIS can compensate for actuator uncertainty and input delays. In Section V, we give our new adaptive ISS result for 3D curve tracking under the polygonal state constraints from Section III. Our new Lyapunov-Krasovskii functional construction helps overcome the challenges of extending our 2D adaptive results [8] to 3D. Our work is mainly a methodological development, rather than a specific real-world application or experiments. However, by combining parameter identification, state constraints, ISS, and delay compensation, our certified performance results are more amenable to the 3D curves in real time marine robotics applications, such as the search for pollution from oil spills. This note is a summary of results from [7], which has proofs of all results to follow.

\section{Review: Nonadaptive 3D CuRve Tracking}

We review the necessary background for the nonadaptive case from [9], to keep our work self contained. Trajectories traced by a pair of particles moving in 3D space were studied in [3], [19]. One particle moves freely, so we call it the free particle. The other is confined to a specified 3D curve. The second particle has locally the shortest distance to the free particle, so we call the second particle the closest point.

Let $\mathbf{r}_{1}$ denote the position of the second particle (at the closest point), $\mathbf{x}_{1}$ the unit tangent vector to the curve at the 
point $\mathbf{r}_{1}, \mathbf{y}_{1}$ a unit normal vector, and $\mathbf{z}_{1}$ a binormal vector. The velocity of the point is in the direction of $\mathbf{x}_{1}$. Let $\mathbf{r}_{2}$ denote the position of the free particle moving at unit speed, $\mathbf{x}_{2}$ the unit tangent vector to the trajectory of its moving center, $\mathbf{y}_{2}$ a corresponding unit normal vector, and $\mathbf{z}_{2}=\mathbf{x}_{2} \times$ $\mathbf{y}_{2}$. With the speed defined by $\frac{d s}{d t}=\alpha$, the time evolutions of the point on the curve and the closest point are [19]

$$
\begin{array}{ll}
\dot{\mathbf{r}}_{1}=\alpha \mathbf{x}_{1} & \dot{\mathbf{r}}_{2}=\mathbf{x}_{2} \\
\dot{\mathbf{x}}_{1}=\alpha \kappa_{n} \mathbf{y}_{1}+\alpha \kappa_{g} \mathbf{z}_{1} & \dot{\mathbf{x}}_{2}=u \mathbf{y}_{2}+v \mathbf{z}_{2} \\
\dot{\mathbf{y}}_{1}=-\alpha \kappa_{n} \mathbf{x}_{1} & \dot{\mathbf{y}}_{2}=-u \mathbf{x}_{2} \\
\dot{\mathbf{z}}_{1}=-\alpha \kappa_{g} \mathbf{x}_{1}, & \dot{\mathbf{z}}_{2}=-v \mathbf{x}_{2},
\end{array}
$$

where $\kappa_{n}$ is the normal curvature, $\kappa_{g}$ is the geodesic curvature, and $u$ and $v$ are steering controls we will choose. We assume that both curvatures are $C^{1}$, bounded and nonpositive valued. The control objective is for $\left|\mathbf{r}_{2}-\mathbf{r}_{1}\right|$ to converge to a desired positive constant, and to control $\mathbf{x}_{2}$ to be aligned with $\mathbf{x}_{1}$, meaning $\mathbf{x}_{1} \cdot \mathbf{x}_{2}= \pm 1$. The state space will be chosen to be a forward invariant set where $\alpha$ is always positive.

We choose the controls $u=a_{1}\left(\mathbf{x}_{1} \cdot \mathbf{y}_{2}\right)+a_{2}\left(\mathbf{y}_{1} \cdot \mathbf{y}_{2}\right)+a_{3}\left(\mathbf{z}_{1}\right.$. $\left.\mathbf{y}_{2}\right)$ and $v=a_{1}\left(\mathbf{x}_{1} \cdot \mathbf{z}_{2}\right)+a_{2}\left(\mathbf{y}_{1} \cdot \mathbf{z}_{2}\right)+a_{3}\left(\mathbf{z}_{1} \cdot \mathbf{z}_{2}\right)$ where the $a_{i}$ 's are functions of the variables $\rho_{1}=\left(\mathbf{r}_{2}-\mathbf{r}_{1}\right) \cdot \mathbf{y}_{1}$ and $\rho_{2}=$ $\left(\mathbf{r}_{2}-\mathbf{r}_{1}\right) \cdot \mathbf{z}_{1}$ and the shape variables $\varphi=\mathbf{x}_{1} \cdot \mathbf{x}_{2}, \beta=\mathbf{y}_{1} \cdot \mathbf{x}_{2}$, and $\gamma=\mathbf{z}_{1} \cdot \mathbf{x}_{2}$ and a steering constant $\mu>0$, namely,

$a_{1}=\mu, a_{2}=-h_{1}^{\prime}\left(\rho_{1}\right)+\frac{\alpha \kappa_{n}}{\varphi}$ and $a_{3}=-h_{2}^{\prime}\left(\rho_{2}\right)+\frac{\alpha \kappa_{g}}{\varphi}$

and where for simplicity we take the penalty functions

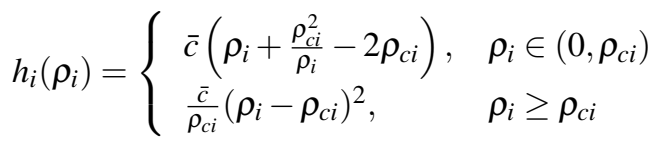

for $i=1,2$ for any tuning constant $\bar{c}>0$ (but see [9] for analogs for more general penalty functions). Then [7] $\alpha=\varphi /\left(1-\kappa_{n} \rho_{1}-\kappa_{g} \rho_{2}\right)$. The $\rho_{c i}$ 's are any desired positive values for $\rho_{1}$ and $\rho_{2}$, so our alignment objective is to achieve UGAS to the equilibrium $\left(\rho_{c 1}, \rho_{c 2}, 1,0,0\right)$ from all initial states in $\mathscr{Z}=\left\{\left(\rho_{1}, \rho_{2}, \varphi, \beta, \gamma\right) \in(0,+\infty)^{2} \times S^{2}: \varphi>0\right\}$. Note that $h_{i}^{\prime}\left(\rho_{i}\right)>0$ (resp., $<0$ ) if $\rho_{i}>\rho_{c i}$ (resp., $\left.<\rho_{c i}\right)$.

Finally, taking the spherical coordinates $(\varphi, \beta, \gamma)=$ $(\cos (\zeta) \cos (\theta),-\sin (\zeta) \cos (\theta), \sin (\theta))$ gives [9]

$$
\begin{aligned}
\dot{\rho}_{1}= & -\sin (\zeta) \cos (\theta) \\
\dot{\zeta}= & -\frac{1}{\cos ^{2}(\theta)}\left[\alpha \kappa_{n} \sin ^{2}(\theta)-h_{1}^{\prime}\left(\rho_{1}\right) \cos (\zeta) \cos (\theta)\right. \\
& \left.+\alpha \kappa_{g} \sin (\theta) \sin (\zeta) \cos (\theta)+\mu \sin (\zeta) \cos (\theta)\right] \\
\dot{\rho}_{2}= & \sin (\theta) \\
\dot{\theta}= & \alpha \kappa_{g} \frac{\sin ^{2}(\zeta)}{\cos (\zeta)}-h_{2}^{\prime}\left(\rho_{2}\right) \cos (\theta)-\mu \cos (\zeta) \sin (\theta) \\
& +\left(-h_{1}^{\prime}\left(\rho_{1}\right)+\frac{\alpha \kappa_{n}}{\cos (\theta) \cos (\zeta)}\right) \sin (\zeta) \sin (\theta)
\end{aligned}
$$

on the state space

$$
\mathscr{X}=(0,+\infty) \times(-\pi / 2, \pi / 2) \times(0,+\infty) \times(-\pi / 2, \pi / 2),
$$

and our UGAS objective will be realized if we prove that (4) is UGAS to its equilibrium $\mathscr{E}=\left(\rho_{c 1}, 0, \rho_{c 2}, 0\right)$ on $\mathscr{X}$. For brevity, we denote the state of (4) by $Y=\left(\rho_{1}, \zeta, \rho_{2}, \theta\right)$.

While it is possible to prove UGAS of (4) to $\mathscr{E}$ using the nonstrict Lyapunov function $V(Y)=$ $-\ln (\cos (\theta))-\ln (\cos (\zeta))+h_{1}\left(\rho_{1}\right)+h_{2}\left(\rho_{2}\right)$ on $\mathscr{X}$ and LaSalle invariance, a key result from [9] is a strict Lyapunov function $U$ for (4), which uses the functions $\lambda(q)=\lambda_{0}\left(q, \rho_{c 1}\right)+\lambda_{0}\left(q, \rho_{c 2}\right)+2 \bar{c} / \min \left\{\rho_{c 1}, \rho_{c 2}\right\}, \Gamma(q)=$ $\Gamma_{0}\left(q, \rho_{c 1}\right)+\Gamma_{0}\left(q, \rho_{c 2}\right)+4 \bar{c} q / \min \left\{\rho_{c 1}, \rho_{c 2}\right\}$, and

$$
\begin{aligned}
\mathscr{L}(q)= & \frac{3}{\mu}[\Gamma(q)+1]\left(1+\left\|\kappa_{n}\right\|_{\infty}+\left\|\kappa_{g}\right\|_{\infty}\right) \\
& +2\left[\frac{1}{\mu} \lambda(q)+\Gamma^{\prime}(q)+1\right], \text { where } \\
\lambda_{0}\left(q, \rho_{c i}\right)= & \frac{2}{\bar{c}^{2} \rho_{c i}^{4}}\left(q+2 \bar{c} \rho_{c i}\right)^{3}+1+0.5 \mu^{2}+\mu \\
\text { and } \Gamma_{0}\left(q, \rho_{c i}\right)= & \frac{18 \bar{c}}{\rho_{c i}} q+\left(\frac{2}{\rho_{c i}}\right)^{4}\left(\frac{9}{\bar{c}^{2}}\right) q^{4} \text { for } i=1,2 .
\end{aligned}
$$

The strict Lyapunov function construction from [9] is:

Theorem 1: The function

$$
\begin{aligned}
U(Y)= & -h_{1}^{\prime}\left(\rho_{1}\right) \sin (\zeta) \cos (\theta) \\
& +h_{2}^{\prime}\left(\rho_{2}\right) \sin (\theta)+\int_{0}^{V(Y)} \mathscr{L}(q) \mathrm{d} q
\end{aligned}
$$

is a strict Lyapunov function for (4) on its state space (5). Therefore, (4) is UGAS to $\mathscr{E}=\left(\rho_{c 1}, 0, \rho_{c 2}, 0\right)$ on $\mathscr{X}$.

The proof of Theorem 1 shows that along all trajectories of (4) in $\mathscr{X}$, we have $U \geq V$ and $\dot{U} \leq-0.5\left[h_{1}^{\prime}\left(\rho_{1}\right) \cos (\zeta)\right]^{2}-$ $\sin ^{2}(\zeta)-0.5\left[h_{2}^{\prime}\left(\rho_{2}\right) \cos (\theta)\right]^{2}-\sin ^{2}(\theta)$. The second inequality is a strict decay property, because its right side is only zero at $\mathscr{E}$. This strictness property will be key for building our adaptive controls and Lyapunov Krasovskii functional for the more difficult input delayed perturbed adaptive 3D tracking problem. The other key ingredient is the following nontrivial extension of the nonadaptive RFIS constructions from [9].

\section{3D ADAPTIVE RFIS}

The rest of this paper summarizes some new results from [7]. In practice, the control gains might not be known. Instead, only an interval $\left(g_{\min }, g_{\max }\right)$ containing the gains, with known constant positive endpoints, may be available. We model this by replacing $u$ and $v$ in (1) by $G u / \hat{G}$ and $G v / \hat{G}$ respectively, where $G \in\left(g_{\min }, g_{\max }\right)$ is an unknown constant, and where $\hat{G}$ is an estimate of $G$. The $\hat{G}$ dynamics will have the state space $\left(g_{\min }, g_{\max }\right)$ and will be such that $\hat{G}(t) \rightarrow G$ for all initial states $\hat{G}(0) \in\left(g_{\min }, g_{\max }\right)$. Equivalently, $G$ is the unknown control gain, and we use the scaled controllers $u_{\text {new }}=u / \hat{G}$ and $v_{\text {new }}=v / \hat{G}$, where $u$ and $v$ are defined in terms of the weights (2) exactly as above. Then similar calculations to the ones in the last section except with the new controls give the augmented 3D adaptive curve tracking system [7]

$$
\begin{aligned}
\dot{\rho}_{1}= & -\sin (\zeta) \cos (\theta) \\
\dot{\zeta}= & -\frac{\cos (\zeta) \kappa_{n}}{1-\kappa_{n} \rho_{1}-\kappa_{g} \rho_{2}} \frac{1}{\cos (\theta)}\left(\frac{G}{\hat{G}}-\cos ^{2}(\theta)\right)+\delta_{1} \\
& -\frac{\alpha \kappa_{g} \sin (\theta) \sin (\zeta)}{\cos (\theta)}+\frac{G}{\hat{G}} \frac{h_{1}^{\prime}\left(\rho_{1}\right) \cos (\zeta)}{\cos (\theta)}-\frac{G}{\hat{G}} \frac{\mu \sin (\zeta)}{\cos (\theta)} \\
\dot{\rho}_{2}= & \sin (\theta) \\
\dot{\theta}= & -\frac{\kappa_{g} \cos ^{2}(\zeta) \cos (\theta)}{1-\kappa_{n} \rho_{1}-\kappa_{g} \rho_{2}}+\frac{G}{\hat{G}} H_{A}(Y) \sin (\zeta) \sin (\theta) \\
& +\left[\frac{G}{\hat{G}} H_{B}(Y) \cos (\theta)\right]-\frac{G \mu \cos (\zeta) \sin (\theta)}{\hat{G}}+\delta_{2} \\
\dot{\hat{G}}= & \left(g_{\text {max }}-\hat{G}\right)\left(\hat{G}-g_{\text {min }}\right) \frac{1}{\hat{G}}\left(\frac{\partial U}{\partial \zeta} \mathscr{A}_{1}+\frac{\partial U}{\partial \theta} \mathscr{A}_{2}\right)
\end{aligned}
$$

on the enlarged state space $\mathscr{X}_{a}=\mathscr{X} \times\left(g_{\min }, g_{\max }\right)$, where $\kappa_{n}$ and $\kappa_{g}$ are functions of $Y$, and where we use the functions

$$
\begin{aligned}
& H_{A}(Y)=\frac{\kappa_{n}}{1-\kappa_{n} \rho_{1}-\kappa_{g} \rho_{2}}-h_{1}^{\prime}\left(\rho_{1}\right) \text { and } \\
& H_{B}(Y)=\frac{\kappa_{g}}{1-\kappa_{n} \rho_{1}-\kappa_{g} \rho_{2}}-h_{2}^{\prime}\left(\rho_{2}\right)
\end{aligned}
$$


and $\mathscr{A}_{1}(Y)=-\left(H_{A}(Y) \cos (\zeta)+\mu \sin (\zeta)\right) / \cos (\theta)$ and $\mathscr{A}_{2}(Y)=-\mu \cos (\zeta) \sin (\theta)+H_{A}(Y) \sin (\zeta) \sin (\theta)+$ $H_{B}(Y) \cos (\theta)$ on the state space (5) and the $\delta_{i}$ 's are unknown measurable essentially bounded real valued functions representing uncertainties (which we later use to capture the effects of delays or control uncertainty). Although update laws such as our dynamics for $\hat{G}$ have appeared in the literature, to the best of our knowledge, none of the existing results can cover the ISS and state constraint results for (7) that we consider here.

We must restrict the sup norms of the $\delta_{i}$ 's to have forward invariance of the state space $\mathscr{X}_{a}$ of (7). To see why this restriction is needed, take the initial state $\left(\rho_{1}(0), \zeta(0), \rho_{2}(0), \theta(0), \hat{G}(0)\right)=\left(2 \rho_{c 1}, 0,2 \rho_{c 2}, 0, G\right) \in$ $\mathscr{X}_{a}$ and the constant disturbance components $\delta_{1}(t)=0$ and $\delta_{2}(t)=-\left\|\kappa_{g}\right\|_{\infty}-g_{\max }\left(h_{1}^{\prime}\left(3 \rho_{c 1}\right)+\left\|\kappa_{n}\right\|_{\infty}+\mu\right) / g_{\min }-$ $\pi /\left(2 \min \left\{\rho_{c 1}, \rho_{c 2}\right\}\right)$, where $\|\cdot\|_{\infty}$ is the sup norm. Since each trajectory of (7) satisfies $\left|\dot{\rho}_{i}(t)\right| \leq 1$ for all $t \geq 0$, we have $\rho_{i}(t) \in\left[\rho_{c i}, 3 \rho_{c i}\right]$ and so also $h_{i}^{\prime}\left(\rho_{i}(t)\right) \geq 0$ for all $t \in\left[0, \min \left\{\rho_{c 1}, \rho_{c 2}\right\}\right]$ for $i=1,2$. Since we assumed that $\kappa_{g}$ is nonpositive valued and $h_{1}^{\prime \prime}\left(\rho_{1}\right) \geq 0$ for all $\rho_{1}>0$, the term in brackets in (7) is nonpositive on $\mathscr{X}_{a}$, so the corresponding trajectory of (7) satisfies $\dot{\theta}(t) \leq\left\|\kappa_{g}\right\|_{\infty}+g_{\max }\left(h_{1}^{\prime}\left(3 \rho_{c 1}\right)+\right.$ $\left.\left\|\kappa_{n}\right\|_{\infty}+\mu\right) / g_{\min }+\delta_{2}(t)=-\pi /\left(2 \min \left\{\rho_{c 1}, \rho_{c 2}\right\}\right)$ for all $t \in$ $\left[0, \min \left\{\rho_{c 1}, \rho_{c 2}\right\}\right]$ for which the trajectory is in $\mathscr{X}_{a}$. Since $\theta(0)=0$, it follows that $\theta(t)$ reaches $-\pi / 2$ by time $t=$ $\min \left\{\rho_{c 1}, \rho_{c 2}\right\}$, so the trajectory exits from $\mathscr{X}_{a}$.

This motivates our search for robustly forwardly invariant sets and corresponding maximal perturbation sets for (7). By robust forward invariance of a subset $\mathscr{S}$ of the state space of a system, with perturbations valued in a set $\mathscr{D}$, we mean that all trajectories of the system starting in $\mathscr{S}$ for all perturbations valued in $\mathscr{D}$ stay in $\mathscr{S}$ for all future times. In [9] we found robustly forwardly invariant sets with maximal perturbation sets for the nonadaptive case, but they do not apply to (7). The adaptive robustly forwardly invariant sets from [8] only apply in 2D, and cannot compensate arbitrarily long input delays or arbitrarily large perturbations as we will do below. Instead, we use the following RFIS construction.
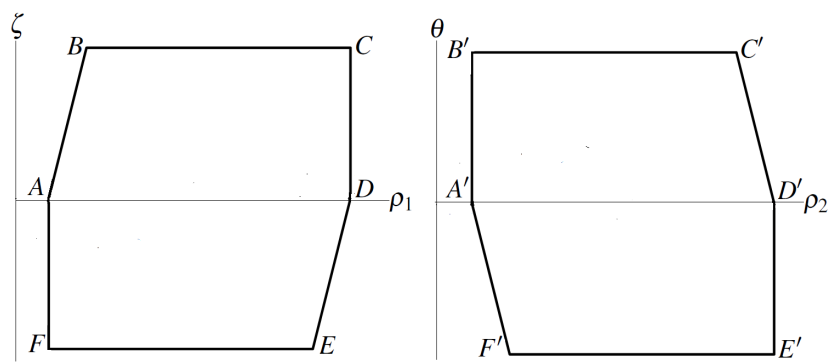

Fig. 1. Hexagononal Regions $H_{1}\left(\rho_{* 1}, \bar{\zeta}, K_{1}\right)$ and $H_{2}\left(\rho_{* 2}, \bar{\theta}, K_{2}\right)$ for Robustly Forwardly Invariant Set $H_{1}\left(\rho_{* 1}, \bar{\zeta}, K_{1}\right) \times H_{2}\left(\rho_{* 2}, \bar{\theta}, K_{2}\right)$

Take any quadruple $\left(\rho_{* 1}, \rho_{* 2}, K_{1}, K_{2}\right)$ of positive constants, with $\rho_{* i} \in\left(0, \rho_{c i}\right)$ for $i=1,2$. Fix any constants $\bar{\zeta} \in(0, \pi / 2)$ and $\bar{\theta} \in(0, \pi / 2)$, and set $\mu^{\sharp}=\mu g_{\min } / g_{\max }$. Let $H_{1}\left(\rho_{* 1}, \bar{\zeta}, K_{1}\right)$ be the closed set in the $\left(\rho_{1}, \zeta\right)$ plane whose boundary is the hexagon having the vertices $A=$ $\left(\rho_{* 1}, 0\right), B=\left(\rho_{* 1}+\bar{\zeta} / \mu^{\sharp}, \bar{\zeta}\right), C=\left(\rho_{* 1}+2 \bar{\zeta} / \mu^{\sharp}+K_{1}, \bar{\zeta}\right)$, $D=\left(\rho_{* 1}+2 \bar{\zeta} / \mu^{\sharp}+K_{1}, 0\right), E=\left(\rho_{* 1}+\bar{\zeta} / \mu^{\sharp}+K_{1},-\bar{\zeta}\right)$, and
$F=\left(\rho_{* 1},-\bar{\zeta}\right)$. Its legs $A B$ and $D E$ have slope $\mu^{\sharp}$, and its other legs are horizontal or vertical. Let $H_{2}\left(\rho_{* 2}, \bar{\theta}, K_{2}\right)$ be the closed set in the $\left(\rho_{2}, \theta\right)$ plane whose boundary is the hexagon with the vertices $A^{\prime}=\left(\rho_{* 2}, 0\right), B^{\prime}=\left(\rho_{* 2}, \bar{\theta}\right), C^{\prime}=$ $\left(\rho_{* 2}+\bar{\theta} /\left(\mu^{\sharp} \cos (\bar{\theta})\right)+K_{2}, \bar{\theta}\right), D^{\prime}=\left(\rho_{* 2}+2 \bar{\theta} /\left(\mu^{\sharp} \cos (\bar{\theta})\right)+\right.$ $\left.K_{2}, 0\right), E^{\prime}=\left(\rho_{* 2}+2 \bar{\theta} /\left(\mu^{\sharp} \cos (\bar{\theta})\right)+K_{2},-\bar{\theta}\right)$, and $F^{\prime}=$ $\left(\rho_{* 2}+\bar{\theta} /\left(\mu^{\sharp} \cos (\bar{\theta})\right),-\bar{\theta}\right)$. Its legs $A^{\prime} F^{\prime}$ and $C^{\prime} D^{\prime}$ have slope $-\cos (\bar{\theta}) \mu^{\sharp}$, and its other legs are horizontal or vertical. See Figure 1. These resemble the hexagons for the nonadaptive case in [9], but are different because we used the bounds on the control gain to tilt the slopes of some of the legs. To specify the hexagon parameters, we first write the $Y$ subdynamics of (7) as

$$
\begin{aligned}
\dot{\rho}_{1} & =-\sin (\zeta) \cos (\theta) \\
\dot{\zeta} & =\mathscr{Q}_{1}^{\mathscr{A}}(Y, \hat{G})+\delta_{1} \\
\dot{\rho}_{2} & =\sin (\theta) \\
\dot{\theta} & =\mathscr{Q}_{2}^{\&}(Y, \hat{G})+\delta_{2} .
\end{aligned}
$$

so $\mathscr{Q}_{2}^{\mathscr{A}}(Y, \hat{G})$ is the sum of the first four terms on the right side of $\dot{\theta}$ in (7) and similarly for $\dot{\zeta}$. Given any constants $M_{i}>0$ for $i=1,2$, we choose the hexagon parameters to satisfy the requirements from:

Lemma 1: There are constants $\bar{\rho}_{* 1} \in\left(0, \rho_{c 1}\right)$ and $\bar{K}_{1}>$ $\rho_{c 1}$ such that for each pair $\left(\rho_{* 1}, K_{1}\right) \in\left(0, \bar{\rho}_{* 1}\right) \times\left(\bar{K}_{1},+\infty\right)$, there exist constants $\mu>0, \rho_{* 2} \in\left(0, \rho_{c 2}\right)$ and $K_{2}>0$ such that the following four conditions hold: (C1) $\mathscr{Q}_{1}^{\mathscr{d}}(Y, \hat{G})+$ $\mu^{\sharp} \sin (\zeta) \cos (\theta)>M_{1}$ for all $\left(\rho_{1}, \zeta\right) \in E D$ and all $\left(\rho_{2}, \theta\right) \in$ $H_{2}\left(\rho_{* 2}, \bar{\theta}, K_{2}\right)$. Also, $\mathscr{Q}_{1}^{\mathscr{A}}(Y, \hat{G})+\mu^{\sharp} \sin (\zeta) \cos (\theta)<-M_{1}$ for all $\left(\rho_{1}, \zeta\right) \in A B$ and all $\left(\rho_{2}, \theta\right) \in H_{2}\left(\rho_{* 2}, \bar{\theta}, K_{2}\right)$. (C2) $\mathscr{Q}_{1}^{\mathscr{A}}(Y, \hat{G})>M_{1}$ for all $\left(\rho_{1}, \zeta\right) \in F E$ and all $\left(\rho_{2}, \theta\right) \in$ $H_{2}\left(\rho_{* 2}, \bar{\theta}, K_{2}\right)$. Also, $\mathscr{Q}_{1}^{\mathscr{A}}(Y, \hat{G})<-M_{1}$ for all $\left(\rho_{1}, \zeta\right) \in$ $B C$ and all $\left(\rho_{2}, \theta\right) \in H_{2}\left(\rho_{* 2}, \bar{\theta}, K_{2}\right)$. (C3) $\mathscr{Q}_{2}^{\mathscr{A}}(Y, \hat{G})+$ $\mu^{\sharp} \cos (\bar{\zeta}) \sin (\theta)>M_{2}$ for all $\left(\rho_{2}, \theta\right) \in A^{\prime} F^{\prime}$ and all $\left(\rho_{1}, \zeta\right) \in$ $H_{1}\left(\rho_{* 1}, \bar{\zeta}, K_{1}\right)$. Also, $\mathscr{Q}_{2}^{\mathscr{A}}(Y, \hat{G})+\mu^{\sharp} \cos (\bar{\zeta}) \sin (\theta)<-M_{2}$ for all $\left(\rho_{2}, \theta\right) \in C^{\prime} D^{\prime}$ and all $\left(\rho_{1}, \zeta\right) \in H_{1}\left(\rho_{* 1}, \bar{\zeta}, K_{1}\right)$. (C4) $\mathscr{Q}_{2}^{\mathscr{A}}(Y, \hat{G})>M_{2}$ for all $\left(\rho_{2}, \theta\right) \in F^{\prime} E^{\prime}$ and all $\left(\rho_{1}, \zeta\right) \in$ $H_{1}\left(\rho_{* 1}, \bar{\zeta}, K_{1}\right)$. Also, $\mathscr{Q}_{2}^{\mathscr{d}}(Y, \hat{G})<-M_{2}$ for all $\left(\rho_{2}, \theta\right) \in B^{\prime} C^{\prime}$ and all $\left(\rho_{1}, \zeta\right) \in H_{1}\left(\rho_{* 1}, \bar{\zeta}, K_{1}\right)$.

See [7] for the proof of Lemma 1. We also set $\Delta_{a}=\min _{\hat{G}}\left\{\left|\mathscr{Q}_{1}^{\mathscr{A}}(Y, \hat{G})+\mu^{\sharp} \sin (\zeta) \cos (\theta)\right|:\left(\rho_{1}, \zeta\right) \in\right.$ $\left.A B \cup E D,\left(\rho_{2}, \theta\right) \in H_{2}\left(\rho_{* 2}, \bar{\theta}, K_{2}\right)\right\}, \Delta_{b}=\min _{\hat{G}}\left\{\left|\mathscr{Q}_{1}^{\mathscr{A}}(Y, \hat{G})\right|:\right.$ $\left.\left(\rho_{1}, \zeta\right) \in F E \cup B C,\left(\rho_{2}, \theta\right) \in H_{2}\left(\rho_{* 2}, \bar{\theta}, K_{2}\right)\right\}, \quad \Delta_{c}=$ $\min _{\hat{G}}\left\{\left|\mathscr{Q}_{2}^{\mathscr{A}}(Y, \hat{G})+\mu^{\sharp} \cos (\bar{\zeta}) \sin (\theta)\right|:\left(\rho_{2}, \theta\right) \in C^{\prime} D^{\prime} \cup\right.$ $\left.A^{\prime} F^{\prime},\left(\rho_{1}, \zeta\right) \in H_{1}\left(\rho_{* 1}, \bar{\zeta}, K_{1}\right)\right\}$, and $\Delta_{d}=\min _{\hat{G}}\left\{\left|\mathscr{Q}_{2}^{\mathscr{A}}(Y, \hat{G})\right|\right.$ : $\left.\left(\rho_{2}, \theta\right) \in B^{\prime} C^{\prime} \cup F^{\prime} E^{\prime},\left(\rho_{1}, \zeta\right) \in H_{1}\left(\rho_{* 1}, \bar{\zeta}, K_{1}\right)\right\}$, where the subscript $\hat{G}$ means that we are also minimizing over all $\hat{G} \in\left[g_{\min }, g_{\max }\right]$. Then conditions (C1)-(C4) of Lemma 1 imply that $\min \left\{\Delta_{a}, \Delta_{b}\right\} \geq M_{1}$ and $\min \left\{\Delta_{c}, \Delta_{d}\right\} \geq M_{2}$ hold.

Next notice that since there is no perturbation in the update law for $\hat{G}$, forward invariance of $H_{1}\left(\rho_{* 1}, \bar{\zeta}, K_{1}\right) \times$ $H_{2}\left(\rho_{* 2}, \bar{\theta}, K_{2}\right) \times\left(g_{\min }, g_{\max }\right)$ for (7) for a given perturbation set $\mathscr{D}$ is equivalent to forward invariance of $H_{1}\left(\rho_{* 1}, \bar{\zeta}, K_{1}\right) \times$ $H_{2}\left(\rho_{* 2}, \bar{\theta}, K_{2}\right)$ for (9) for the same perturbation set for each solution $\hat{G}$ of the update law in (7). Hence, to prove our robust forward invariant results, we focus on the dynamics (9). In terms of the constants $\bar{\Delta}_{\zeta}=\min \left\{\Delta_{a}, \Delta_{b}\right\}$ and $\bar{\Delta}_{\theta}=$ $\min \left\{\Delta_{c}, \Delta_{d}\right\}$, we have the following consequence from [7]: 
Theorem 2: Let $\left(M_{1}, M_{2}, \bar{\zeta}, \bar{\theta}, \rho_{* 1}, \rho_{* 2}, K_{1}, K_{2}, \mu\right)$ be such that the requirements $(\mathrm{C} 1)-(\mathrm{C} 4)$ from Lemma 1 hold. Then: (a) For each $C^{1}$ solution $\hat{G}:[0,+\infty) \rightarrow\left(g_{\min }, g_{\max }\right)$ of the update law and all constants $\delta_{* 1} \in\left(0, \bar{\Delta}_{\zeta}\right)$ and $\delta_{* 2} \in\left(0, \bar{\Delta}_{\theta}\right)$, the set $H_{1}\left(\rho_{* 1}, \bar{\zeta}, K_{1}\right) \times H_{2}\left(\rho_{* 2}, \bar{\theta}, K_{2}\right)$ is robustly forwardly invariant for (9) with the disturbance set $\mathscr{D}=\left[-\delta_{* 1}, \delta_{* 1}\right] \times$ $\left[-\delta_{* 2}, \delta_{* 2}\right]$. (b) For each constant $\delta_{+}>\bar{\Delta}_{\zeta}\left(\right.$ resp., $\left.>\bar{\Delta}_{\theta}\right)$, there exist a point $Y \in \partial\left(H_{1}\left(\rho_{* 1}, \bar{\zeta}, K_{1}\right) \times H_{2}\left(\rho_{* 2}, \bar{\theta}, K_{2}\right)\right)$ and a solution $\hat{G}$ such that the trajectory for (9) starting at $Y$ for one of the constant perturbations $\pm\left(\delta_{+}, 0\right)$ (resp., $\pm\left(0, \delta_{+}\right)$) immediately exits $H_{1}\left(\rho_{* 1}, \bar{\zeta}, K_{1}\right) \times H_{2}\left(\rho_{* 2}, \bar{\theta}, K_{2}\right)$.

Remark 1: As an analog to the 2D case from [6], our conditions can be satisfied by a nested increasing sequence of hexagonal product regions that fill $\mathscr{X}=(0,+\infty) \times$ $(-\pi / 2, \pi / 2) \times(0,+\infty) \times(-\pi / 2, \pi / 2)$. This can be done by picking $\bar{\zeta}$ and $\bar{\theta}$ to be close enough to $\pi / 2$, reducing the $\rho_{* i}$ 's, and finally increasing the $K_{i}$ 's and $\mu$ so the hexagons converge to bigger and bigger rectangles. Therefore, for any given compact subset $S_{0} \subseteq \mathscr{X}$, we can choose our hexagon parameters such that $S_{0} \subseteq H_{1}\left(\rho_{* 1}, \bar{\zeta}, K_{1}\right) \times H_{2}\left(\rho_{* 2}, \bar{\theta}, K_{2}\right)$, and then all trajectories of the $Y$ dynamics starting in $S_{0}$ stay in $H_{1}\left(\rho_{* 1}, \bar{\zeta}, K_{1}\right) \times H_{2}\left(\rho_{* 2}, \bar{\theta}, K_{2}\right)$ under the maximum perturbation box of the theorem. This can ensure predictable tolerance and safety bounds for all trajectories starting in $S_{0}$, without imposing any regularity at the boundary of $S_{0}$. We cannot replace our hexagons with arbitrary bounded polygons, such as boxes [7].

Remark 2: Part (b) of Theorem 2 gives maximality of the bounds $\bar{\Delta}_{\zeta}$ and $\bar{\Delta}_{\theta}$ for maintaining the robust forward invariance, which is analogous to the maximal disturbance bound in [6], [8] for maintaining robust forward invariance in 2D tracking. On the other hand, given any desired disturbance bound $\bar{M}$, we can make $H_{1}\left(\rho_{* 1}, \bar{\zeta}, K_{1}\right) \times H_{2}\left(\rho_{* 2}, \bar{\theta}, K_{2}\right)$ robustly forwardly invariant under disturbances that are bounded by $\bar{M}$, so we can compensate arbitrarily large perturbations. This follows because Lemma 1 ensures that we can tune the hexagon parameters to make the constants from $\Delta_{a}, \Delta_{b}, \Delta_{c}$, and $\Delta_{d}$ as large as we want. This contrasts with the 2D adaptive result from [8], where the penalty functions in the control have the usual form $h(\rho)=\bar{c}\left(\rho+\rho_{c}^{2} / \rho-2 \rho_{c}\right)$ from [19], and where there was a finite upper bound on the allowable perturbations, so the results of this section are stronger than [8] even in the 2D case.

\section{DELAY COMPENSATION VIA RFIS}

The $\delta_{i}$ 's in (7) represent controller uncertainty, and our analysis leads to upper bounds on the allowable actuator errors. They can capture the effects of delays in the $Y=$ $\left(\rho_{1}, \zeta, \rho_{2}, \theta\right)$ values entering our controls, and then we can compute bounds on the allowable delays that give robust forward invariance. We found these delay bounds in [9] for the $3 \mathrm{D}$ nonadaptive case. In this section, we find the delays bounds in the more challenging adaptive case, which will give a way to compensate arbitrarily long input delays.

Let $\|f\|_{\mathscr{I}}$ denote the supremum of any function $f$ on any set $\mathscr{I} \subseteq$ domain $(f)$. Let $h_{1}, h_{2}, \bar{\zeta}, \bar{\theta}, \mu, \delta_{*}=\left(\delta_{* 1}, \delta_{* 2}\right)$, and $H_{1}\left(\rho_{* 1}, \bar{\zeta}, K_{1}\right) \times H_{2}\left(\rho_{* 2}, \bar{\theta}, K_{2}\right)$ satisfy the requirements from
Theorem 2. Throughout what follows, all upper bounds are over points or trajectories in this product set unless otherwise indicated, and we assume that that there are no delays in the update law and that $\kappa_{n}$ and $\kappa_{g}$ are negative constants; see [7] for more general cases. Then our controls are the constant $a_{1}=\mu$, and the nonconstant controls $a_{2}$ and $a_{3}$ in (2), which are functions of $\rho=\left(\rho_{1}, \rho_{2}\right)$. We assume that there is a constant delay $\tau_{i}>0$ in the values of $\rho$ in $a_{i}$ for $i=2,3$. Choosing the constants

$$
\begin{aligned}
& \mathscr{M}_{2}=\frac{1}{g_{\min }}\left(\|\left. h_{1}^{\prime \prime}\right|_{\left[\rho_{* 1}, \rho_{* 1}+K_{1}+2\right]}+\kappa_{n}^{2}+\left|\kappa_{n} \kappa_{g}\right|\right) \text { and } \\
& \mathscr{M}_{3}=\frac{1}{g_{\min }}\left(\|\left. h_{2}^{\prime \prime}\right|_{\left[\rho_{* 2}, \rho_{* 2}+K_{2}+2\right]}+\kappa_{g}^{2}+\left|\kappa_{n} \kappa_{g}\right|\right)
\end{aligned}
$$

gives $\left|a_{i}\left(\rho\left(t-\tau_{i}\right)\right)-a_{i}(\rho(t))\right| \leq \mathscr{M}_{i} \tau_{i} g_{\text {min }}$ for $i=2,3$ (using the fact that the $\dot{\rho}_{i}$ 's are bounded by 1 and the Mean Value Theorem, where the terms involving the $h_{i}^{\prime \prime}$ 's capture the effects of the terms $-h_{i}^{\prime}\left(\rho_{i}\right)$, and assuming without loss of generality that $\mu^{\sharp} \geq \bar{\zeta}$ and $\left.\mu^{\sharp} \cos (\bar{\theta}) \geq \overline{\bar{\theta}}\right)$. We write the undelayed adaptive system (7) as

$$
\dot{Y}^{\sharp}=\mathscr{F}\left(Y^{\sharp}\right)+\left(0, \delta_{1}, 0, \delta_{2}, 0\right)^{\top}
$$

for a suitable function $\mathscr{F}$, where $Y^{\sharp}=\left(\rho_{1}, \zeta, \rho_{2}, \theta, \hat{G}\right)$. On the other hand, replacing the controls $a_{i}$ in (2) by the delayed versions $a_{i}\left(\rho\left(t-\tau_{i}\right)\right)$ for $i=2,3$ and using the spherical change of coordinates as above, we get the perturbed adaptive input delayed system

$$
\dot{Y}^{\sharp}=\mathscr{F}\left(Y^{\sharp}\right)+\left(0, \Xi_{\zeta}\left(Y_{t}^{\sharp}\right)+\tilde{\delta}_{1}, 0, \Xi_{\theta}\left(Y_{t}^{\sharp}\right)+\tilde{\delta}_{2}, 0\right)^{\top},
$$

where $\tilde{\delta}_{1}$ and $\tilde{\delta}_{2}$ represent additive uncertainties, $Y_{t}^{\sharp}(s)=$ $Y^{\sharp}(s+t)$ for $-\max \left\{\tau_{2}, \tau_{3}\right\} \leq s \leq 0$, and

$$
\begin{aligned}
\Xi_{\zeta}\left(Y_{t}^{\sharp}\right)= & -\frac{\cos (\zeta) G}{\cos (\theta) \hat{G}}\left[a_{2}\left(\rho\left(t-\tau_{2}\right)\right)-a_{2}(\rho(t))\right] \\
\Xi_{\theta}\left(Y_{t}^{\sharp}\right)= & \frac{G}{\hat{G}}\left(\sin (\theta) \sin (\zeta)\left[a_{2}\left(\rho\left(t-\tau_{2}\right)\right)-a_{2}(\rho(t))\right]\right. \\
& \left.+\cos (\theta)\left[a_{3}\left(\rho\left(t-\tau_{3}\right)\right)-a_{3}(\rho(t))\right]\right),
\end{aligned}
$$

where we used the fact that $\hat{G}$ in (7) is valued in $\left(g_{\min }, g_{\max }\right) \subseteq(0, \infty)$ and omit the dependence of $\theta$ and $\zeta$ on $t$. This gives $\left|\Xi_{\zeta}\left(Y_{t}^{\sharp}\right)\right| \leq G \mathscr{M}_{2} \tau_{2} / \cos (\bar{\theta})$ and $\left|\Xi_{\theta}\left(Y_{t}^{\sharp}\right)\right| \leq G\left(\sin (\bar{\theta}) \sin (\bar{\zeta}) \mathscr{M}_{2} \tau_{2}+\mathscr{M}_{3} \tau_{3}\right) \quad$ on $H_{1}\left(\rho_{* 1}, \bar{\zeta}, K_{1}\right) \times H_{2}\left(\rho_{* 2}, \bar{\theta}, K_{2}\right)$. Also, (12) is the special case of (11) with the choices $\left(\delta_{1}(t), \delta_{2}(t)\right)=$ $\left(\Xi_{\zeta}\left(Y_{t}^{\sharp}\right)+\tilde{\delta}_{1}(t), \Xi_{\theta}\left(Y_{t}^{\sharp}\right)+\tilde{\delta}_{2}(t)\right)$, and they satisfy $\left\|\delta_{i}\right\|_{\infty} \leq\left\|\tilde{\delta}_{i}\right\|_{\infty}+\max \left\{\tau_{2}, \tau_{3}\right\} G \mathscr{M}_{4}$ for $i=1,2$, where

$$
\mathscr{M}_{4}=\left(\frac{1}{\cos (\bar{\theta})}+\sin (\bar{\zeta}) \sin (\bar{\theta})\right) \mathscr{M}_{2}+\mathscr{M}_{3} \text {. }
$$

Hence, by Theorem $2, H_{1}\left(\rho_{* 1}, \bar{\zeta}, K_{1}\right) \times H_{2}\left(\rho_{* 2}, \bar{\theta}, K_{2}\right)$ is robustly forward invariant for (12) for all $\left(\tilde{\delta}_{1}, \tilde{\delta}_{2}\right)$ 's satisfying $\left\|\tilde{\delta}_{i}\right\|_{\infty}+\max \left\{\tau_{2}, \tau_{3}\right\} G \mathscr{M}_{4}<\delta_{* i}$ for $i=1,2$. This shows [7]:

Theorem 3: Let $H_{1}\left(\rho_{* 1}, \bar{\zeta}, K_{1}\right) \times H_{2}\left(\rho_{* 2}, \bar{\theta}, K_{2}\right), \mu, \delta_{* 1}$, and $\delta_{* 2}$ satisfy the above requirements, and the curvatures $\kappa_{n}$ and $\kappa_{g}$ be constant and negative. Choose any constants $\delta_{* * i}>0$ and any positive constants $\tau_{2}$ and $\tau_{3}$ such that

$$
\delta_{* * i} \leq \delta_{* i}-\max \left\{\tau_{2}, \tau_{3}\right\} G \mathscr{M}_{4} \text { for } i=1,2 .
$$

Then $H_{1}\left(\rho_{* 1}, \bar{\zeta}, K_{1}\right) \times H_{2}\left(\rho_{* 2}, \bar{\theta}, K_{2}\right) \times\left(g_{\min }, g_{\max }\right)$ is robustly forwardly invariant for the adaptive input delayed curve tracking dynamics (12) for all disturbances $\left(\tilde{\delta}_{1}, \tilde{\delta}_{2}\right)$ valued in $\mathscr{D}=\left[-\boldsymbol{\delta}_{* * 1}, \boldsymbol{\delta}_{* * 1}\right] \times\left[-\boldsymbol{\delta}_{* * 2}, \boldsymbol{\delta}_{* * 2}\right]$.

Formula (15) requires subtracting terms from the $\delta_{* i}$ 's to get perturbation bounds that hold under the input delays. This 
reduces the admissible disturbance set. On the other hand, we can choose the hexagon parameters to get arbitrarily small reductions in the $\delta_{* i}$ 's and compensate arbitrarily long input delays, as follows. Assume for simplicity that the input delays only occur in the speed measurements $\alpha=$ $\varphi /\left(1-\kappa_{n} \rho_{1}-\kappa_{g} \rho_{2}\right)$ in the controls, and that $\kappa_{n}$ and $\kappa_{g}$ are negative constants. This produces the perturbed delayed adaptive system (12) as before, except with $a_{2}\left(\rho\left(t-\tau_{2}\right)\right)$ and $a_{3}\left(\rho\left(t-\tau_{3}\right)\right)$ replaced by

$$
\begin{aligned}
& \bar{a}_{2}\left(Y_{t}\right)= \\
& -h_{1}^{\prime}\left(\rho_{1}(t)\right)+\frac{\kappa_{n} \cos \left(\zeta\left(t-\tau_{2}\right)\right) \cos \left(\theta\left(t-\tau_{2}\right)\right)}{\cos (\zeta(t)) \cos (\theta(t))\left(1-\kappa_{n} \rho_{1}\left(t-\tau_{2}\right)-\kappa_{g} \rho_{2}\left(t-\tau_{2}\right)\right)} \\
& \bar{a}_{3}\left(Y_{t}\right)= \\
& -h_{2}^{\prime}\left(\rho_{2}(t)\right)+\frac{\kappa_{g} \cos \left(\zeta\left(t-\tau_{3}\right)\right) \cos \left(\theta\left(t-\tau_{3}\right)\right)}{\cos (\zeta(t)) \cos (\theta(t))\left(1-\kappa_{n} \rho_{1}\left(t-\tau_{3}\right)-\kappa_{g} \rho_{2}\left(t-\tau_{3}\right)\right)}
\end{aligned}
$$

respectively. With this replacement, the new terms in (12) capturing the inputs delays have the bounds

$$
\begin{aligned}
& \left|\Xi_{\zeta}\left(Y_{t}^{\sharp}\right)\right| \leq \frac{G\left(\tau_{2}+1\right) \mathscr{B}\left(\kappa_{n}, \kappa_{g}, \bar{\zeta}, \bar{\theta}\right)}{\cos (\bar{\theta}) g_{\min }} \text { and } \\
& \left|\Xi_{\theta}\left(Y_{t}^{\sharp}\right)\right| \leq \frac{2 G\left(\max \left\{\tau_{2}, \tau_{3}\right\}+1\right) \mathscr{B}\left(\kappa_{n}, \kappa_{g}, \bar{\zeta}, \bar{\theta}\right)}{g_{\min }}, \text { where } \\
& \mathscr{B}\left(\kappa_{n}, \kappa_{g}, \bar{\zeta}, \bar{\theta}\right)=\frac{2 \max \left\{\left|\kappa_{n}\right|,\left|\kappa_{g}\right|\right\}+\max \left\{\kappa_{n}^{2}, \kappa_{g}^{2}\right\}+\kappa_{n} \kappa_{g}}{\cos (\bar{\zeta}) \cos (\bar{\theta})}
\end{aligned}
$$

because the terms in the formulas (10) for $\mathscr{M}_{2}$ and $\mathscr{M}_{3}$ involving the $h_{i}^{\prime \prime}$ 's are no longer needed and $\left|\dot{\rho}_{i}\right| \leq 1$ everywhere. Then we reason similarly to the above arguments and replace the upper bound (15) on the allowable $\tilde{\delta}_{i}$ 's by

$$
\delta_{* * i}^{\text {new }}=\delta_{* i}-\left[\frac{2 G\left(\max \left\{\tau_{2}, \tau_{3}\right\}+1\right) \mathscr{B}\left(\kappa_{n}, \kappa_{g}, \bar{\zeta}, \bar{\theta}\right)}{\cos (\bar{\theta}) g_{\min }}\right]
$$

for $i=1,2$. It follows that $\mathscr{S}=H_{1}\left(\rho_{* 1}, \bar{\zeta}, K_{1}\right) \times$ $H_{2}\left(\rho_{* 2}, \bar{\theta}, K_{2}\right) \times\left(g_{\min }, g_{\max }\right)$ is robustly forwardly invariant for $(12)$ when its disturbances $\left(\tilde{\delta}_{1}, \tilde{\delta}_{2}\right)$ are valued in $\left[-\delta_{* * 1}^{\text {new }}, \delta_{* * 1}^{\text {new }}\right] \times\left[-\delta_{* * 2}^{\text {new }}, \delta_{* * 2}^{\text {new }}\right]$, if the requirements of Theorem 2 hold. Moreover, for any constant $\varepsilon \in(0,1)$, we get $\delta_{* * i}^{\text {new }} \geq(1-\varepsilon) \delta_{* i}$ for $i=1,2$ when

$$
\begin{aligned}
& 2 \max \left\{\left|\kappa_{n}\right|,\left|\kappa_{g}\right|\right\}+2 \max \left\{\kappa_{n}^{2}, \kappa_{g}^{2}\right\} \\
& \leq \frac{\varepsilon \min \left\{\delta_{* 1}, \delta_{* 2}\right\} \cos (\bar{\zeta}) \cos ^{2}(\bar{\theta}) g_{\min }}{2 g_{\max }\left(\max \left\{\tau_{2}, \tau_{3}\right\}+1\right)},
\end{aligned}
$$

because (19) implies that the term in brackets in (18) has the bound $\varepsilon \delta_{* i}$ for $i=1,2$.

This gives the following tracking algorithm for any positive constant delays $\tau_{2}$ and $\tau_{3}$ in the speed measurement $\alpha$ and any negative constant choices of $\kappa_{n}$ and $\kappa_{g}$. First, pick any constants $\bar{\zeta} \in(0, \pi / 2), \bar{\theta} \in(0, \pi / 2)$, and $\varepsilon \in(0,1)$. Then, select the hexagon parameters that make $\min \left\{\boldsymbol{\delta}_{* 1}, \boldsymbol{\delta}_{* 2}\right\}$ big enough for (19) to hold. This is possible by Remark 1. We conclude that $\mathscr{S}=H_{1}\left(\rho_{* 1}, \bar{\zeta}, K_{1}\right) \times H_{2}\left(\rho_{* 2}, \bar{\theta}, K_{2}\right) \times$ $\left(g_{\min }, g_{\max }\right)$ is robustly forwardly invariant for (12) with disturbances $\left(\tilde{\delta}_{1}, \tilde{\delta}_{2}\right)$ valued in $\mathscr{D}_{\varepsilon}=\left[-(1-\varepsilon) \delta_{* 1},(1-\varepsilon) \delta_{* 1}\right] \times$ $\left[-(1-\varepsilon) \delta_{* 2},(1-\varepsilon) \delta_{* 2}\right]$. This shows that we can allow arbitrarily long time delays with arbitrarily small reductions in the set of admissible values for the control disturbances.

\section{ISS FOR DELAYED ADAPTIVE SYSTEM}

We now leverage our RFIS results to prove our ISS result. We first recall some relevant definitions. For any constant $\tau \geq 0$ and any subset $\mathscr{S}$ of a Euclidean space, let $\mathscr{C}_{[-\tau, 0]}(\mathscr{S})$ denote the set of all continuous functions $\phi:[-\tau, 0] \rightarrow \mathscr{S}$.
We use the standard classes $\mathscr{K}_{\infty}$ and $\mathscr{K} \mathscr{L}$ of comparison functions [4]. Consider any subset $\mathscr{S}$ of any Euclidean space and any point $\mathscr{E}_{0} \in \mathscr{S}$. A modulus with respect to $\left(\mathscr{E}_{0}, \mathscr{S}\right)$ is any function $\Lambda: \mathscr{S} \rightarrow[0,+\infty)$ that is zero at $\mathscr{E}_{0}$, positive at all other points in $\mathscr{S}$, and radially unbounded in the following sense: For each constant $K>0$, there is a constant $\delta_{K}>0$ such that $\Lambda(x) \geq K$ for all $x \in \mathscr{S}$ that satisfy $\operatorname{dist}(x$, boundary $(\mathscr{S})) \leq \delta_{K}$ or $|x|_{\mathscr{E}_{0}} \geq 1 / \delta_{K}$. Here $|x|_{\mathscr{E}_{0}}=\left|x-\mathscr{E}_{0}\right|$ is the distance to the equilibrium.

Consider any system $\dot{Z}(t)=\mathscr{G}\left(t, Z_{t}, \delta(t)\right)$ with a constant delay $\tau \geq 0$, the state space $\mathscr{S}$, measurable essentially bounded disturbances $\delta$ valued in some subset $\mathscr{D}$ of a Euclidean space, and an equilibrium $\mathscr{E}_{0} \in \mathscr{S}$ (meaning $\mathscr{G}\left(t, \mathscr{E}_{0}, 0\right)=0$ for all $t \geq 0$, where we also use $\mathscr{E}_{0}$ to mean the function in $\mathscr{C}_{[-\tau, 0]}(\mathscr{S})$ that is identically equal to $\left.\mathscr{E}_{0}\right)$. Here $Z_{t}(r)=Z(t+r)$ for $-\tau \leq r \leq 0$, and we assume that all dynamics have a well defined flow map. The system is called input-to-state stable (ISS) on $\mathscr{S}$ (for $\mathscr{D}$ and $\mathscr{E}_{0}$ ) provided there are functions $\beta \in \mathscr{K} \mathscr{L}$ and $\gamma \in \mathscr{K}_{\infty}$ and a modulus $\Lambda$ with respect to $\left(\mathscr{E}_{0}, \mathscr{S}\right)$ such that $|Z(t)|_{\mathscr{E}_{0}} \leq$ $\beta\left(\left\|\Lambda\left(Z_{0}(r)\right)\right\|_{[-\tau, 0]}, t\right)+\gamma\left(\|\delta\|_{[0, t]}\right)$ for all initial functions $Z_{0} \in \mathscr{C}_{[-\tau, 0]}(\mathscr{S})$, all disturbances $\delta$ valued in $\mathscr{D}$, all $t \geq 0$, and the trajectory $Z(t)$ for the system for $\delta$ starting at $Z_{0}$.

Recall from Theorem 3 that we must restrict the input delays to get robust forward invariance. To find delay bounds that give ISS results on each of our product sets $\mathscr{S}=$ $H_{1}\left(\rho_{* 1}, \bar{\zeta}, K_{1}\right) \times H_{2}\left(\rho_{* 2}, \bar{\theta}, K_{2}\right) \times\left(g_{\min }, g_{\max }\right)$, we set

$\bar{M}=g_{\max }^{2}\left(2 \sin ^{2}(\bar{\zeta}) \sin ^{2}(\bar{\theta})+\frac{1}{\cos ^{2}(\bar{\theta})}\right) \mathscr{M}_{2}^{2}+2 g_{\max }^{2} \mathscr{M}_{3}^{2}$ and $\bar{\tau}=\frac{\bar{c}_{1} \bar{c}_{2}}{2 \sqrt{\bar{M} L}}$

where the $\mathscr{M}_{i}$ 's are from (10), the constant $L>0$ satisfies $|((d / d \zeta) U(Y),(d / d \theta) U(Y))| \leq L|Y|_{\mathscr{E}}$ on $\mathscr{S}$ for our function $U$ from (6), $\bar{\zeta} \in(0, \pi / 2)$ and $\bar{\theta} \in(0, \pi / 2)$ are from our definition of $H_{1}\left(\rho_{* 1}, \bar{\zeta}, K_{1}\right) \times H_{2}\left(\rho_{* 2}, \bar{\theta}, K_{2}\right)$, and $\bar{c}_{1}$ and $\bar{c}_{2}$ are chosen such that $\dot{U}(Y) \leq-\bar{c}_{1} U(Y)$ and $\bar{c}_{2}|Y|_{\mathscr{E}}^{2} \leq U(Y)$ hold along all trajectories of the unperturbed system on $\mathscr{S}$. See [7] for the proof that such $\bar{c}_{i}$ 's exist. In [7], we prove:

Theorem 4: Let $H_{1}\left(\rho_{* 1}, \bar{\zeta}, K_{1}\right) \times H_{2}\left(\rho_{* 2}, \bar{\theta}, K_{2}\right), \quad \tau_{2} \in$ $(0, \bar{\tau}), \tau_{3} \in(0, \bar{\tau}), \delta_{* * 1}$, and $\delta_{* * 2}$ satisfy the requirements from Theorem 3. Then the perturbed adaptive $3 \mathrm{D}$ tracking dynamics (12) is ISS on $\mathscr{S}=H_{1}\left(\rho_{* 1}, \bar{\zeta}, K_{1}\right) \times H_{2}\left(\rho_{* 2}, \bar{\theta}, K_{2}\right) \times$ $\left(g_{\min }, g_{\max }\right)$ for the disturbance set $\mathscr{D}=\left[-\delta_{* * 1}, \boldsymbol{\delta}_{* * 1}\right] \times$ $\left[-\delta_{* * 2}, \delta_{* * 2}\right]$ and the equilibrium $\mathscr{E}^{a}=\left(\rho_{c 1}, 0, \rho_{c 2}, 0, G\right)$.

Sketch of Proof: Take the function

$$
\begin{aligned}
& V^{\sharp \sharp}\left(Y_{t}, \hat{G}_{t}\right)= \\
& V^{\sharp}(Y(t), \hat{G}(t))+(\bar{J}+\bar{\mu}) \int_{t-\max \left\{\tau_{2}, \tau_{3}\right\}}^{t} \int_{s}^{t}|Y(r)|_{\mathscr{E}}^{2} \mathrm{~d} r \mathrm{~d} s,
\end{aligned}
$$

where

$$
\begin{aligned}
V^{\sharp}(Y, \hat{G})= & R\left(U(Y)+\int_{G}^{\hat{G}} \frac{\ell-G}{\left(\ell-g_{\min }\right)\left(g_{\max }-\ell\right)} \mathrm{d} \ell\right) \\
& +(\hat{G}-G)^{2}+(\zeta-\theta)(\hat{G}-G), \\
\bar{J}= & \bar{M}\left(\frac{R L^{2}}{\bar{c}_{1} \bar{c}_{2}}+\frac{2 g_{\max }}{c_{0}+c_{1}}\right) \max \left\{\tau_{2}, \tau_{3}\right\}
\end{aligned}
$$

the constant $\bar{\mu}>0$ is such that $(\bar{J}+\bar{\mu}) \max \left\{\tau_{2}, \tau_{3}\right\}<$ $R \bar{c}_{1} \bar{c}_{2} / 4$, the positive constants $c_{o}$ and $c_{1}$ can be chosen such that there exists a constant $\bar{G}>0$ such that $\max \left\{|\dot{\hat{G}}(Y, \hat{G})|,\left|\mathscr{Q}_{1}^{\mathscr{A}}(Y, G)\right|,\left|\mathscr{Q}_{2}^{\mathscr{A}}(Y, G)\right|\right\} \leq \bar{G}|Y|_{\mathscr{E}}, \mathscr{A}_{1}(Y) \geq$ 
$c_{o}-\bar{G}|Y|_{\mathscr{E}}$ and $\mathscr{A}_{2}(Y) \leq-c_{1}+\bar{G}|Y|_{\mathscr{E}}$ hold along all trajectories of the system contained in $\mathscr{S}=H_{1}\left(\rho_{* 1}, \bar{\zeta}, K_{1}\right) \times$ $H_{2}\left(\rho_{* 2}, \bar{\theta}, K_{2}\right) \times\left(g_{\min }, g_{\max }\right)$ (where $\mathscr{Q}_{1}^{\mathscr{A}}$ and $\mathscr{Q}_{2}^{\mathscr{A}}$ are from (9)), and $R>0$ is any constant such that

$$
\begin{aligned}
& R \geq \frac{2}{\bar{c}_{2}} \max \left\{1, \frac{\bar{H}}{\bar{c}_{1}}, \frac{c_{0}+c_{1}}{2 \bar{c}_{1} g_{\max }}\right\}, \text { where } \\
& \bar{H}=2 \bar{G}+\frac{2 g_{\max } \bar{G}^{2}}{c_{0}+c_{1}}\left(4+\frac{2 g_{\max }}{g_{\min }}\right)^{2}
\end{aligned}
$$

and such that

$$
\max \left\{\tau_{2}, \tau_{3}\right\}<\sqrt{\frac{R \bar{c}_{1}^{2} \bar{c}_{2}^{2}\left(c_{0}+c_{1}\right)}{4 \bar{M}\left[R L^{2}\left(c_{0}+c_{1}\right)+2 g_{\max } \bar{c}_{1} \bar{c}_{2}\right]}} .
$$

Such an $R$ exists because the limit of the right side of (24) as $R \rightarrow+\infty$ is our bound $\bar{\tau}$ from (20). The function $V^{\sharp}$ admits a function $\bar{\gamma} \in \mathscr{K}_{\infty}$ such that $\dot{V}^{\sharp} \leq$ $-0.5 \alpha^{b}\left(Y^{\sharp}\right)+\bar{J} \int_{t-\max \left\{\tau_{2}, \tau_{3}\right\}}^{t}|Y(r)|_{\mathscr{E}}^{2} \mathrm{~d} r+\bar{\gamma}(|\tilde{\delta}|)$ along all trajectories of (12) for all disturbances valued in $\mathscr{D}$, where $\alpha^{b}\left(Y^{\sharp}\right)=0.5 R \bar{c}_{1} \bar{c}_{2}|Y|_{\mathscr{E}}^{2}+(\hat{G}-G)^{2}\left(c_{0}+c_{1}\right) /\left[2 g_{\max }\right]$. Then $V^{\sharp \sharp}$ is a Lyapunov-Krasovskii functional for (12) when the disturbance is zero, and it admits a positive definite function $\alpha_{0}$ such that $\dot{V}^{\sharp \sharp} \leq-\alpha_{0}\left(V^{\sharp \sharp}\right)+\bar{\gamma}(|\tilde{\delta}|)$ along all trajectories of (12) for all disturbances $\delta$ valued in $\mathscr{D}$. This is weaker than an ISS Lyapunov-Krasovskii decay estimate because $V^{\sharp \sharp}$ is unbounded on the bounded set $\mathscr{S}$. However, we can choose $\alpha_{0}$ such that $\lim _{s \rightarrow+\infty} \alpha_{0}(s)>0$, so this leads to the ISS result. See [7] for more details.

Remark 3: We can relax our conditions on $\kappa_{n}$ and $\kappa_{g}$ to the requirements that they are bounded, $C^{1}$, nowhere zero, and time invariant, and this makes our approach amenable to a broader class of 3D curves. This can be done by restricting the state space so that the denominator $1-\kappa_{n} \rho_{1}-\kappa_{g} \rho_{2}$ of $\alpha$ is positive throughout the state space. See [7] for more details. The work [10] gave a scaling method for proving UGAS under arbitrarily long input delays. We can use a similar approach to prove ISS for (12), under arbitrarily long input delays when the delays only enter $\alpha$ and the curvatures are negative constants of small enough absolute value. This is because if the delayed control components are $\bar{a}_{2}$ and $\bar{a}_{3}$ as defined in (16), then we can replace $\bar{M}$ from (20) by a new formula $\bar{M}_{a}$ that converges to 0 when $\kappa_{n} \rightarrow 0^{-}$and $\kappa_{g} \rightarrow 0^{-}$, since the terms involving the $h_{i}^{\prime \prime}$ 's in the formulas for $\mathscr{M}_{2}$ and $\mathscr{M}_{3}$ in (10) are no longer necessary. Moreover, the constants $\bar{c}_{1}, \bar{c}_{2}$, and $L$ can be chosen independently of $\kappa_{n}$ and $\kappa_{g}$ if the curvatures lie in some known compact interval. We conclude that the new delay bound $\bar{\tau}=\bar{c}_{1} \bar{c}_{2} /\left(2 \sqrt{\bar{M}_{a}} L\right)$ converges to $+\infty$ as $\kappa_{n} \rightarrow 0^{-}$and $\kappa_{g} \rightarrow 0^{-}$, so there is a tradeoff between the maximal allowable input delays and the magnitudes of the curvatures [7].

\section{Conclusions}

Curve tracking for marine robots under input delays, actuator uncertainty, unknown control gains, and state constraints is challenging. We obtained new adaptive ISS tracking and parameter identification results for 3D curve tracking using a new Lyapunov-Krasovskii functional design, and a robust forward invariance approach that leads to predictable tolerance and safety bounds under polygonal state constraints. By tuning parameters for the polygons, we proved robust forward invariance under arbitrarily long input delays in an important case where the delays only enter the speed measurements. This is totally different from prediction, reduction, and other delay compensation approaches. Tuning the parameters also leads to ISS under arbitrarily large perturbations. We believe that our robust forward invariance approach may also prove useful in other applications, such as coordinated tracking by teams of marine robots.

\section{REFERENCES}

[1] Z. Artstein. Linear systems with delayed controls: A reduction. IEEE Trans. Automat. Control, 27(4):869-879, 1982.

[2] Y. Han, R. de Callafon, J. Cortes, and J. Jaffe. Dynamic modeling and pneumatic switching control of a submersible drogue. In Proc. International Conf. Informatics in Control, Automation and Robotics, pages 92-97, Funchal, Madeira, Portugal, 2010.

[3] E. Justh and P.S. Krishnaprasad. Natural frames and interacting particles in three dimensions. In Proc. IEEE Conf. Decision and Control and European Control Conf., pages 2841-2846, Seville, Spain, 2005.

[4] H. Khalil. Nonlinear Systems, Third Edition. Prentice-Hall, Upper Saddle River, New Jersey, 2002.

[5] M. Krstic. Delay Compensation for Nonlinear, Adaptive, and PDE Systems. Birkhauser, Boston, MA, 2009.

[6] M. Malisoff, F. Mazenc, and F. Zhang. Stability and robustness analysis for curve tracking control using input-to-state stability. IEEE Trans. Automat. Control, 57(5):1320-1326, 2012.

[7] M. Malisoff and F. Zhang. Adaptive three-dimensional curve tracking control under control uncertainty and state constraints. Preprint. http://www.math.lsu.edu/ malisoff/research.html.

[8] M. Malisoff and F. Zhang. Adaptive control for planar curve tracking under controller uncertainty. Automatica, 49(5):1411-1418, 2013.

[9] M. Malisoff and F. Zhang. Robustness of a class of three-dimensional curve tracking control laws under time delays and polygonal state constraints. In Proc. American Control Conf., pages 5710-5715, Washington, DC, 2013.

[10] F. Mazenc and M. Malisoff. Asymptotic stabilization for feedforward systems with delayed feedbacks. Automatica, 49(3):780-787, 2013.

[11] F. Mazenc, M. Malisoff, and Z. Lin. Further results on input-to-state stability for nonlinear systems with delayed feedbacks. Automatica, 44(9):2415-2421, 2008.

[12] S. Mukhopadhyay, C. Wang, S. Bradshaw, S. Maxon, M. Patterson, and F. Zhang. Controller performance of marine robots in reminiscent oil surveys. In Proc. IEEE/RSJ International Conf. on Intelligent Robots and Systems, pages 1766-1771, Vilamoura, Portugal, 2012.

[13] M. Ouimet and J. Cortes. Distributed estimation of internal wave parameters via inter-drogue distances. In Proc. IEEE Conf. Decision and Control, pages 2433-2438, Maui, HI, 2012.

[14] C. Samson. Control of chained systems: Application to path-following and time-varying point-stabilization of mobile robots. IEEE Trans. Automat. Control, 40(1):64-77, 1995.

[15] S. Shatara and X. Tan. An efficient, time-of-flight-based underwater acoustic ranging system for small robotic fish. IEEE J. Oceanic Engineering, 35(4):837-846, 2010.

[16] X. Tan. Autonomous robotic fish as mobile sensor platforms: Challenges and potential solutions. Marine Technology Society J., 45(4):31-40, 2011.

[17] X. Xiang, L. Lapierre, C. Liu, and B. Jouvencel. Path tracking: combined path following and trajectory tracking for autonomous underwater vehicles. In Proc. IEEE/RSJ International Conf. on Intelligent Robots and Systems, pages 3558-3563, San Francisco, CA, 2011.

[18] F. Zhang, D.M. Fratantoni, D. Paley, J. Lund, and N.E. Leonard. Control of coordinated patterns for ocean sampling. International $J$. Control, 80(7):1186-1199, 2007.

[19] F. Zhang, E. Justh, and P.S. Krishnaprasad. Curve tracking and obstacle avoidance using gyroscopic control. In A. Johann, H-P. Kruse, F. Rupp, and S. Schmitzi, editors, Recent Trends in Dynamical Systems. Proceedings of a Conference in Honor of Jürgen Scheurle, Springer Proceedings in Mathematics and Statistics, Vol. 35, pages 417-446. Springer Basel, New York, 2013.

[20] B. Zhou, Z. Lin, and G. Duan. Stabilization of linear systems with input delay and saturation - a parametric Lyapunov equation approach. International J. Robust Nonlinear Control, 20(13):1502-1519, 2010. 